\title{
o 154 \\ Material strategies and considerations for serologic testing of global infectious diseases
}

\author{
Jessica E. Manning, Patrick E. Duffy, Dominic Esposito, and \\ Kaitlyn Sadtler*(i)
}

The SARS-CoV-2 pandemic has brought to light multiple considerations when approaching infectious diseases on the global level. These range from diagnostic platforms, to therapeutics, and prevention agents. In this article, we focus on the engineering platforms and considerations when applying serologic assays to multiple geographic locations, climates with varying endemic virus repertoires, and different laboratory and clinical resource settings. Serologic assays detect antibodies that react against viral proteins, suggesting prior infection and correlative of an increased likelihood of immunity to future infection. As these assays are focused on the human immune response to a pathogen, and humans are variable, there are a number of important engineering steps to optimize assay performance, from sample collection, to assay execution and data analysis. Moving forward, a global approach to infectious disease detection and prevention is necessary to prevent the spread of future viruses with pandemic potential.

\section{Introduction}

In the context of SARS-CoV-2, we have witnessed rapid development and implementation of various therapeutics and diagnostics to combat the pandemic. However, these diagnostics are often centered around European and North American testing markets, ignoring the different biologic variables at place in varying countries across the world. A number of considerations in material development must be evaluated to have success in all continents, which will be crucial for accurate understanding and control of SARS-CoV-2, along with other emergent infectious diseases (Figure 1).

\section{Cold chain considerations for sample acquisition}

One limit of biomedical research and therapeutics is the cold chain. The need for an uninterrupted temperature-controlled collection, processing, and transport pathway to ensure sample or therapeutic stability can be highly restraining when it comes to long distances or resource-scarce settings.

This translates to sample collection and analysis for diagnostic and infection tracking data. Viral swabs and sampling often require cold storage prior to detection of viral genomic RNA; although the use of RNA preservatives added at time of sample collection can remove required cold chain without significant RNA degradation but eliminates the opportunity to culture the virus (i.e., Zymo Research, QIAGEN). The sample must then transit to a laboratory capable of viral RNA extraction and polymerase chain reaction (PCR), which may be several hours to days' drive away in resource-scarce settings. In the case of evaluating for the presence of viral variants, there may be only one national laboratory in a country equipped with sequencing capabilities. ${ }^{1}$ However, simplification of viral detection through multiple available rapid tests, including loop-mediated isothermal amplification- and probebased RNA detection and lateral-flow antigen (protein) detection kits, has expanded the ability to diagnose SARS-CoV-2 in constrained health care systems at low costs. Point-of-care detection kits with shelf stable components overcome a multitude of obstacles and could serve as a referral to more sensitive laboratory-based tests, or as a standalone in locations where access to laboratory tests is limited.

\footnotetext{
Jessica E. Manning, Laboratory of Malaria and Vector Research, National Institute of Allergy and Infectious Diseases, National Institutes of Health, Cambodia; jessica.manning@nih.gov

Patrick E. Duffy, Laboratory of Malaria Immunology and Vaccinology, National Institute of Allergy and Infectious Diseases, National Institutes of Health, USA; patrick.duffy@nih.gov Dominic Esposito, Protein Expression Laboratory, NCI RAS Initiative, Frederick National Laboratory for Cancer Research, USA; dominic.esposito@nih.gov Kaitlyn Sadtler, National Institute of Biomedical Imaging and Bioengineering, National Institutes of Health, USA; kaitlyn.sadler@nih.gov

${ }^{*}$ Corresponding author

doi:10.1557/s43577-021-00167-4
} 


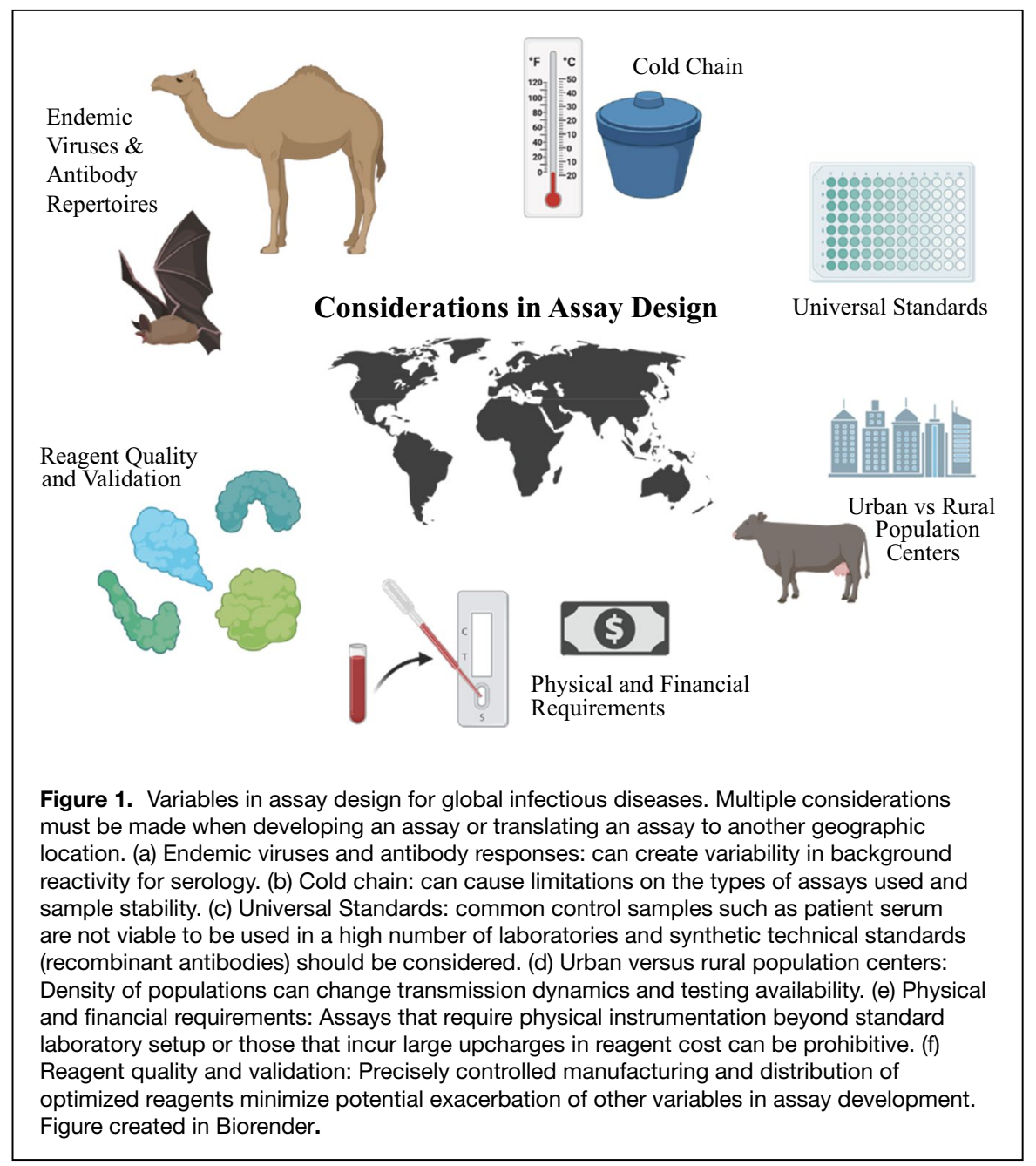

With respect to antibody detection assays that look for the presence of anti-SARS-CoV-2 antibodies in a patient's blood, sample collection method can greatly alter sample stability. Venipuncture blood draws that result in the collection of serum or plasma are not shelf stable and are subject to contamination and bacterial growth if not stored in air-tight sterile storage containers. Serum and plasma samples are also more prone to acting as a reservoir of infectious agents such as HIV and hepatitis. In order to circumvent the need for venipuncture, volumetric serum sampling through subcutaneous and capillary-based blood draw have been developed to allow for home sampling. ${ }^{2}$ While these sampling devices allow for avoidance of venipuncture blood draw that requires a trained phlebotomist, there is still concern regarding sample stability and cost. An alternative to liquid blood samples is dried blood collection. ${ }^{3,4}$ These methods include dried blood spots and engineered solutions that collect precise blood volumes. In the most simplistic form, similar to that most often used in resource-scarce settings for malarial diagnostics, dried blood can be collected on filter paper that is then dried out, and stable at room temperature for weeks, and years when frozen. In this case, the only supplies required for blood sampling are a lancet or needle, an alcohol swab or similar material for fingertip skin sterilization, and the blood sampling device such as filter paper. However, the possible drawback of this approach used widely in resource-constrained settings for tropical diseases is a high degree of variability in sensitivity. ${ }^{3}$ Newer technologies are proving to be more reliable and offer a future for mail-in and room temperature sample collection.

\section{Antigen validation, verification, and evaluation}

In the context of serologic tests, there are multiple approaches to analytics, mainly the rapid lateralflow based assays, and the standard laboratory analysis, enzyme-linked immunosorbent assays (ELISAs), to detect antibodies generated by the patient against specific proteins of the virus. ${ }^{5-9}$ Multiple ELISAs have been developed for detection of antibodies against SARSCoV-2, including those that utilize the nucleocapsid protein $(\mathrm{N})$, the spike protein (S), and more specifically the receptor binding domain of the spike protein (RBD). ${ }^{4}$ Each of these antigens can be engineered to provide an optimal signal to noise ratio on serologic assays, as well as optimal production for materials synthesis.

A variety of factors can contribute to antigen performance, including production hosts, purification processes, and storage/handling considerations. S and RBD proteins are heavily glycosylated and have complex folding, making them amenable only to eukaryotic production for the most part. Glycan patterns can vary from host to host, making it vital that proteins are compared consistently from batch to batch and investigator to investigator to obtain reliable results. In the case of $\mathrm{S}$ protein, the natural trimeric structure is required for high-sensitivity serologic tests - to avoid the complexity of the membrane-spanning region usually responsible for this trimerization, investigators have artificially introduced trimers using a foldon domain from bacteriophage T4. ${ }^{10}$ This produces functional trimers of $\mathrm{S}$, but their stability is likely lower than the natural membrane-bound form, requiring careful analytics of proteins to ensure a consistently high level of functional trimer. For RBD, it has been shown that alterations in purification tags can lead to a significant alteration in ELISA sensitivity, again requiring careful comparison of results between 
experiments using different antigen design. ${ }^{11}$ Finally, careful attention to overall protein quality is essential for any proteins used as serology assay antigens. In many cases, lack of quality or batch-to-batch reproducibility of proteins can lead to failed assays. All proteins generated need to be carefully quality controlled with regard to such factors as aggregation, monodispersity, and purity. To control for batch-to-batch variability, processes such as differential scanning fluorimetry ("thermal shift assays") can be used to ensure that past and present assays are not impacted by differences in the proteins themselves. These techniques can also be used to verify that proteins can be frozen and thawed for use in assays, or if they need to be single-use aliquots to ensure consistent quality.

Additionally, certain antigens can be produced in nonmammalian culture systems with high yield and high purity, making them attractive for laboratories with lower access to funding that makes the more complex antigen production pipelines impractical. ${ }^{12}$

Specific to the SARS-CoV-2 virus and the human immune response to infection, $\mathrm{N}$ antibodies appear rapidly in the infection timeline, but fade within six months of disease onset, making this antigen subject to false negatives. ${ }^{13-16}$ RBD antibodies can take time to develop in comparison to antibodies against the full spike protein, making RBD-only assays also subject to missing positives. The full spike protein can have higher background readings from non-specific binding but can be optimized to minimize false positives. Furthermore, regions that may be subject to variant mutations (e.g., RBD in B.1.351 and P1 variants of SARS-CoV-2) can inhibit antibody binding to RBD, and as such are more subject to mutation-induced signal loss, though at the time of writing this manuscript current variants do not alter serologic assay performance. ${ }^{17}$ Ultimately, a combination of multiple antigens is required for detection of seropositivity. Utilization of antigens that are not present in a vaccine formulation but present in viral infection (e.g., N protein for S-based vaccines) can be used to differentiate between vaccine-induced seropositivity and infectioninduced seropositivity.

\section{Endemic virus repertoire and its effect on test specificity}

Any reference to the sensitivity and specificity of an assay must be completed within the population in which the assay is implemented. Most commonly, assay development occurs in areas with large funding resources for research and development and access to investors to further develop those technologies, such as North America and Europe; however, the populations present in these geographic areas are not subject to exposure to diseases that are more common in other climates.

For example, mainland China and Southeast Asia have the highest abundance and diversity of bats, which are known to carry zoonotic diseases including coronaviruses. Horseshoe bats (Rhinolophus spp.) are considered the reservoir host for SARS-CoV-2, SARS-CoV (responsible for the 2003 outbreak), and other related bat viruses such as RaTG13 that belong to the Sarbecovirus subgenus of betacoronaviruses. ${ }^{18-20}$ Recent reports of sarbecoviruses, a subgenus of Coronoviridae, highly homologous to SARS-CoV-2 in horseshoe bats that are found in Japan (96\% homologous and sequence identity of $81.47 \%$ ), Thailand (96\% sequence identity), and Cambodia (92.6\% sequence identity) in 2013 and 2010, respectively. ${ }^{18,21,22}$ This is of clinical importance in serological testing because in Southeast Asia, as well as more rural provinces in Yunnan Province in southern China, the agricultural practice of bat guano farming is popular and exposes these populations at a much higher frequency to bats than elsewhere. One serosurvey performed after the 2003 SARS-CoV-1 outbreak showed 2.7\% seropositivity to SARS-CoV-1 in Yunnan farmers without history of travel. ${ }^{23}$ Given that guano farming is done via the construction of artificial roostings near personal domiciles or at cave entries, it is plausible to consider increased coronavirus exposure in these populations as they typically do not wear personal protective gear to collect these nutrient-rich fecal droppings that they then sell to farmers. ${ }^{24,25}$ Contemporary surveys of bats in mainland Asia and Southeast Asia reveal high levels of homology between SARS-CoV-2 and circulating virus populations in bats. ${ }^{22,26}$ While published serosurveys are lacking in Southeast Asia, we would anticipate higher than expected seroreactivity in these populations compared to their North American or European counterparts. Without proper region-specific optimization of assays in serosurveys, public health decision-making may be misinformed on the burden of SARS-CoV-2 disease in-country.

Furthermore, in other parts of the globe different coronaviruses are prevalent and endemic. Northern Africa and the Middle-East harbor reservoirs of Middle East Respiratory Syndrome Coronavirus (MERS-CoV) in camels that are indigenous to these climates. When SARS-CoV-2 ELISA assays optimized in the United States were tested against pre-pandemic blood samples from West Africa (Mali), a large false positive rate was detected, variant upon the antigen type with $\mathrm{N}$ resulting in higher false positive rates than $\mathrm{S}$ and RBD antigens. ${ }^{27}$ Reactivity to $\mathrm{S}$ antigen of other coronaviruses did not correlate well with SARS-CoV-2 reactivity, so other factors may contribute in Mali. For example, false positivity on commercial SARS-CoV-2 serological assays was related to elevated anti-Plasmodium IgG levels in Nigeria, ${ }^{28}$ and a recent study suggests acute malaria infection may generate crossreactive antibodies to the terminal sialic acids on $\mathrm{S}$ antigen. ${ }^{29}$ When adjusting for the specific population, these assays could be utilized in large-scale seroprevalence studies to accurately measure disease spread in a population.

Samples collected from the United States showed an increased signal intensity in ELISA for seasonal coronaviruses $\mathrm{HCoV}-\mathrm{OC} 43$ and $\mathrm{HCoV}-\mathrm{HKU} 1$ when the individual was SARS-CoV-2 seropositive, suggesting cross-reactivity of anti-S SARS-CoV-2 antibodies with OC43 and HKU1 spike proteins. This could potentially confound future serologic studies of these season or "common cold" coronaviruses due to cross-reactivity. ${ }^{30,31}$ 


\section{Standardization of controls}

Beyond the assays themselves, equally as important are the controls used to quantifiably standardize results. Historically, this has been done through the imprecise evaluation of sensitivity and specificity based off convalescent clinical control samples and seronegative control samples derived from blood donors. While critical for interpretation of the assay in different populations, in order to fully standardize assays and allow for cross-comparisons, there is a need to adopt a long-lasting and widely available standard set of controls. Initial assays of antibody prevalence were developed in the $1940 \mathrm{~s}$ in the context of hematology (Coombs tests), ${ }^{32}$ almost 40 years before the development of the first recombinant monoclonal antibodies in the $1980 \mathrm{~s} .{ }^{33}$ Monoclonal antibodies can be present in specific known concentrations that allow for generation of a sigmoidal regression through a dilution curve that will produce the upper- and lower limits of detection based on a standardized reagent. Adoption of these engineered constant reagents will allow for direct comparison of various assays without the need for human samples which are limited in their distribution potential and of unknown absolute concentration.

\section{Conclusions}

There are multiple steps of materials engineering that need to be considered when developing serologic assays for global infectious diseases. Main considerations include cold chain limitations, ease of sample acquisition, and assay controls and validation specific to the regional population's exposure to coronaviruses. There are facets of each stage that can be approached with engineering solutions, from development of more stable reagents through chemical and protein engineering, to low-cost systems for scalable technologies. Some technologies already exist in the laboratory and merely need to be adopted in a widespread fashion. All aspects of sample acquisition to analysis will need to be considered for prevention of far-reaching infectious diseases, and support nations that bear the brunt of endemic viruses due to indigenous zoonotic vector species.

\section{Funding}

This research was supported in part by the Intramural Research Program of the NIH, including the National Institute of Biomedical Imaging and Bioengineering and the National Institute of Allergy and Infectious Diseases. This project has been funded in whole or in part with federal funds from the National Cancer Institute, National Institutes of Health, under Contract No. HHSN261200800001E.

\section{Conflict of interest}

$\mathrm{KS}$ is an inventor on a provisional patent application that is related to serologic assays for SARS-CoV-2.

\section{Disclaimer}

The content of this publication does not necessarily reflect the views or policies of the Department of Health and Human Services, nor does mention of trade names, commercial products, or organizations imply endorsement by the US Government. The NIH, its officers, and employees do not recommend or endorse any company, product, or service.

\section{Open Access}

This article is licensed under a Creative Commons Attribution 4.0 International license, which permits use, sharing, adaptation, distribution and reproduction in any medium or format, as long as you give appropriate credit to the original author(s) and the source, provide a link to the Creative Commons license, and indicate if changes were made. The images or other third party material in this article are included in the article's Creative Commons license, unless indicated otherwise in a credit line to the material. If material is not included in the article's Creative Commons license and your intended use is not permitted by statutory regulation or exceeds the permitted use, you will need to obtain permission directly from the copyright holder. To view a copy of this license, visit http:// creativecommons.org/licenses/by/4.0/.

\section{References}

1. J.E. Manning, J.A. Bohl, S. Lay, S. Chea, L. Sovann, Y. Sengdoeurn, S. Heng, C. Vuthy, K. Kalantar, V. Ahyong, Rapid metagenomic characterization of a case of imported COVID-19 in Cambodia. BioRxiv (2020). https://doi.org/10.1101/2020.03.02.968818 2. K.J. Williams, J. Lutman, C. McCaughey, S.K. Fischer, Assessment of low volume sampling technologies: Utility in nonclinical and clinical studies. Bioanalysis 13, 679 (2021) 3. P.W. Smit, I. Elliott, R.W. Peeling, D. Mabey, P.N. Newton, An overview of the clinical use of filter paper in the diagnosis of tropical diseases. Am. J. Trop. Med. Hyg. 90, 195 (2014) 4. C. Klumpp-Thomas, H. Kalish, M. Drew, S. Hunsberger, K. Snead, M.P. Fay, J. Mehalko, A. Shunmugavel, V. Wall, P. Frank, J.-P. Denson, M. Hong, G. Gulten, S. Messing, J. Hicks, S. Michael, W. Gillette, M.D. Hall, M.J. Memoli, D. Esposito, K. Sadtler, Standardization of ELISA protocols for serosurveys of the SARS-CoV-2 pandemic using clinical and at-home blood sampling. Nat. Commun. 12, 113 (2021)

5. M. Ainsworth, M. Andersson, K. Auckland, J.K. Baillie, E. Barnes, S. Beer, A. Beveridge, S. Bibi, L. Blackwell, M. Borak, Performance characteristics of five immunoassays for SARS-CoV-2: A head-to-head benchmark comparison. Lancet Infect. Dis. 20, e298 (2020) 6. A. Bryan, G. Pepper, M.H. Wener, S.L. Fink, C. Morishima, A. Chaudhary, K.R. Jerome, P.C. Mathias, A.L. Greninger, Performance characteristics of the Abbott Architect SARS-CoV-2 IgG assay and seroprevalence in Boise, Idaho. J. Clin. Microbiol. 58, e00941-20 (2020)

7. R.H. Jacobson, Validation of serological assays for diagnosis of infectious diseases. Rev. Sci. Tech. Off. Int. Epiz. 17, 469 (1998)

8. A. Petherick, Developing antibody tests for SARS-CoV-2. Lancet 395, 1101 (2020) 9. J.D. Whitman, J. Hiatt, C.T. Mowery, B.R. Shy, R. Yu, T.N. Yamamoto, U. Rathore, G.M. Goldgof, C. Whitty, J.M. Woo, Evaluation of SARS-CoV-2 serology assays reveals a range of test performance. Nat. Biotechnol. 38, 1174 (2020)

10. D. Wrapp, N. Wang, K.S. Corbett, J.A. Goldsmith, C.-L. Hsieh, 0. Abiona, B.S. Graham, J.S. McLellan, Cryo-EM structure of the 2019-nCoV spike in the prefusion conformation. Science 367, 1260 (2020)

11. J. Mehalko, M. Drew, K. Snead, J.-P. Denson, V. Wall, T. Taylor, K. Sadtler, S. Messing, W. Gillette, D. Esposito, Improved production of SARS-CoV-2 spike receptorbinding domain (RBD) for serology assays. Protein Expr. Purif. 179, 105802 (2020) 12. T. Djukic, M. Mladenovic, D. Stanic-Vucinic, J. Radosavljevic, K. Smiljanic, L. Sabljic, M. Devic, D. Cujic, T. Vasovic, A. Simovic, Expression, purification and immunological characterization of recombinant nucleocapsid protein fragment from SARSCoV-2. Virology 557, 15 (2021)

13. D.F. Gudbjartsson, G.L. Norddahl, P. Melsted, K. Gunnarsdottir, H. Holm, E. Eythorsson, A.O. Arnthorsson, D. Helgason, K. Bjarnadottir, R.F. Ingvarsson, Humoral immune response to SARS-CoV-2 in Iceland. New Engl. J. Med. 383, 1724 (2020) 
14. A.T. Huang, B. Garcia-Carreras, M.D. Hitchings, B. Yang, L.C. Katzelnick, S.M. Rattigan, B.A. Borgert, C.A. Moreno, B.D. Solomon, I. Rodriguez-Barraquer, A systematic review of antibody mediated immunity to coronaviruses: Antibody kinetics, correlates of protection, and association of antibody responses with severity of disease. MedRxiv (2020). https://doi.org/10.1101/2020.04.14.20065771

15. Q.-X. Long, B.-Z. Liu, H.-J. Deng, G.-C. Wu, K. Deng, Y.-K. Chen, P. Liao, J.-F. Qiu, Y. Lin, X.-F. Cai, Antibody responses to SARS-CoV-2 in patients with COVID-19. Nat. Med. 26, 845 (2020)

16. Q.-X. Long, X.-J. Tang, Q.-L. Shi, Q. Li, H.-J. Deng, J. Yuan, J.-L. Hu, W. Xu, Y. Zhang, F.-J. Lv, Clinical and immunological assessment of asymptomatic SARS-CoV-2 infections. Nat. Med. 26, 1200 (2020)

17. C. Klumpp-Thomas, H. Kalish, J. Hicks, J. Mehalko, M. Drew, M.J. Memoli, M.D. Hall, D. Esposito, K. Sadtler, D614G spike variant does not alter IgG, IgM, or IgA spike seroassay performance. J. Infect. Dis. (2020). https://doi.org/10.1093/infdis/jiaa743 18. S. Murakami, T. Kitamura, J. Suzuki, R. Sato, T. Aoi, M. Fujii, H. Matsugo, H. Kamiki, H. Ishida, A. Takenaka-Uema, Detection and characterization of bat Sarbecovirus phylogenetically related to SARS-CoV-2, Japan. Emerg. Infect. Dis. 26, 3025 (2020)

19. A.G. Wrobel, D.J. Benton, P. Xu, C. Roustan, S.R. Martin, P.B. Rosenthal, J.J. Skehel, S.J. Gamblin, SARS-CoV-2 and bat RaTG13 spike glycoprotein structures inform on virus evolution and furin-cleavage effects. Nat. Struct. Mol. Biol. 27, 763 (2020) 20. P. Zhou, X.-L. Yang, X.-G. Wang, B. Hu, L. Zhang, W. Zhang, H.-R. Si, Y. Zhu, B. Li, C.-L. Huang, A pneumonia outbreak associated with a new coronavirus of probable bat origin. Nature 579, 270 (2020)

21. V. Hul, D. Delaune, E.A. Karlsson, A. Hassanin, P.O. Tey, A. Baidaliuk, F. Gámbaro, V.T. Tu, L. Keatts, J. Mazet, A novel SARS-CoV-2 related coronavirus in bats from Cambodia. BioRxiv (2021).

22. S. Wacharapluesadee, C.W. Tan, P. Maneeorn, P. Duengkae, F. Zhu, Y. Joyjinda, T. Kaewpom, W.N. Chia, W. Ampoot, B.L. Lim, Evidence for SARS-CoV-2 related coronaviruses circulating in bats and pangolins in Southeast Asia. Nat. Commun. 12, 1430 (2021) 23. N. Wang, S.-Y. Li, X.-L. Yang, H.-M. Huang, Y.-J. Zhang, H. Guo, C.-M. Luo, M. Miller, G. Zhu, A.A. Chmura, Serological evidence of bat SARS-related coronavirus infection in humans, China. Virol Sin. 33, 104 (2018)

24. N.Q. Huong, N.T.T. Nga, N.V. Long, B.D. Luu, A. Latinne, M. Pruvot, N.T. Phuong, L.T.V. Quang, V.V. Hung, N.T. Lan, Coronavirus testing indicates transmission risk increases along wildlife supply chains for human consumption in Vietnam, 20132014. PLoS One 15, e0237129 (2020)

25. A. Lacroix, V. Duong, V. Hul, S. San, H. Davun, K. Omaliss, S. Chea, A. Hassanin, $W$. Theppangna, S. Silithammavong, Genetic diversity of coronaviruses in bats in Lao PDR and Cambodia. Infect. Genet. Evol. 48, 10 (2017)

26. H. Zhou, X. Chen, T. Hu, J. Li, H. Song, Y. Liu, P. Wang, D. Liu, J. Yang, E.C. Holmes, A novel bat coronavirus closely related to SARS-CoV-2 contains natural insertions at the S1/S2 cleavage site of the spike protein. Curr. Biol. 30, 2196 (2020)

27. J. Woodford, I. Sagara, J. Kwan, A. Zeguime, I. Zaidi, O. Attaher, M. Kone, J.Y. Doritchamou, J.P. Renn, M. Maiga, SARS-CoV-2 seroassay optimization and performance in a population with high background reactivity in Mali. MedRxiv (2021). https://doi. org/10.1101/2021.03.08.21252784

28. L.C. Steinhardt, F. Ige, N.C. Iriemenam, S.M. Greby, Y. Hamada, M. Uwandu, M. Aniedobe, K.A. Stafford, A.I. Abimiku, N. Mba, Cross-reactivity of two SARS-CoV-2 serological assays in a malaria-endemic setting. J. Clin. Microbiol. 59, e0051421 (2021)

29. S. Lapidus, F. Liu, A. Casanovas-Massana, Y. Dai, J.D. Huck, C. Lucas, J. Klein, R.B. Filler, M.S. Strine, M. Sy, A.B. Deme, A.S. Badiane, B. Dieye, I.M. Ndiaye, Y. Diedhiou, A.M. Mbaye, C.T. Diagne, I. Vigan-Womas, A. Mbengue, B.D. Sadio, M.M. Diagne, A.J. Moore, K. Mangou, F. Diallo, S.D. Sene, M.N. Pouye, R. Faye, B. Diouf, N. Nery, F. Costa, M. Reis, M.C. Muenker, D.Z. Hodson, Y. Mbarga, B.Z. Katz, J.R. Andrews, M. Campbell, A. Srivathsan, K. Kamath, E. Baum-Jones, O. Faye, A.A. Sall, J.C.Q. Vélez, M. Cappello, M. Wilson, C. Ben-Mamoun, F.A. Somé, R.K. Dabiré, C.E.E. Moukoko, J.B. Ouédraogo, Y. Boum, J. Shon, D. Ndiaye, A. Wisnewski, S. Parikh, A. Iwasaki, C.B. Wilen, A.I. Ko, A.M. Ring, A.K. Bei, Plasmodium infection induces cross-reactive antibodies to carbohydrate epitopes on the SARS-CoV-2 Spike protein. MedRxiv. (2021). https:// doi.org/10.1101/2021.05.10.21256855

30. J. Hicks, C. Klumpp-Thomas, H. Kalish, A. Shunmugavel, J. Mehalko, J.-P. Denson, K.R. Snead, M. Drew, K.S. Corbett, B.S. Graham, Serologic cross-reactivity of SARSCoV-2 with endemic and seasonal Betacoronaviruses. J. Clin. Immunol. 41, 906 (2021) 31. S. Khan, R. Nakajima, A. Jain, R.R. De Assis, A. Jasinskas, J.M. Obiero, O. Adenaiye, S. Tai, F. Hong, D.K. Milton, Analysis of serologic cross-reactivity between common human coronaviruses and SARS-CoV-2 using coronavirus antigen microarray. BioRxiv (2020). https://doi.org/10.1101/2020.03.24.006544

32. A.S. Wiener, A New Test (Blocking Test) for Rh Sensitization, in Rhesus Haemolytic Disease (Springer, New York, 1944), p. 81
33. S.L. Morrison, Transfectomas provide novel chimeric antibodies. Science $\mathbf{2 2 9}$ $1202(1985)$

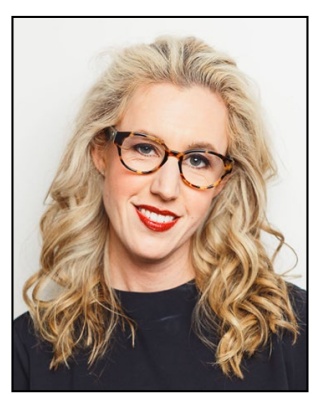

Jessica E. Manning is an infectious diseases physician-scientist with the National Institute of Allergy and Infectious Diseases (NIAID) at the National Institutes of Health (NIH). Since 2008, Manning has lived and worked in Africa and Southeast Asia with a research focus on mosquito-borne diseases like dengue and malaria. Currently, she is an assistant clinical investigator also serving as Science Attaché at the US Embassy Phnom Penh and head of the NIAID International Center of Excellence in Research Cambodia, a collaborative research partnership with the Cambodian Ministry of Health. Manning can be reached by email at jessica.manning@nih.gov.

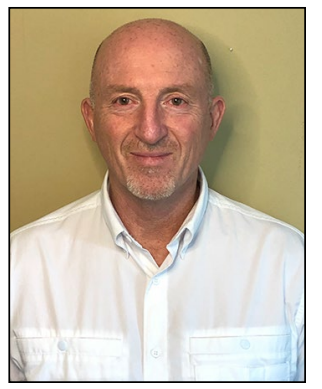

Patrick E. Duffy is the chief of the Laboratory of Malaria Immunology and Vaccinology (LMIV) at the National Institute of Allergy and Infectious Diseases/National Institutes of Health (NIAID/NIH) LMIV developed the first malaria transmissionblocking vaccines to enter field trials. He previously served as Malaria Program Director at Seattle Biomedical Research Institute, affiliate professor of global health at the University of Washington, and principal investigator of the Fogarty-funded East African regional training program for young scientists. He received his MD from Duke University, his internal medicine training at Walter Reed, and postdoctoral training at NIH. Duffy can be reached by email at patrick.duffy@nih.gov.

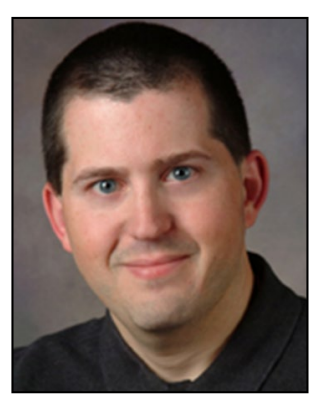

Dominic Esposito is the director of the Protein Expression Laboratory (PEL) at the Frederick National Laboratory for Cancer Research (FNLCR). The PEL produces proteins for structural biology and drug discovery in the NCI RAS Initiative and invents and develops new technologies for protein production. Esposito received his $\mathrm{PhD}$ degree in biochemistry and biophysics from the Johns Hopkins University Bloomberg School, carried out postdoctoral research at the National Institutes of Health, and worked as a staff scientist at Life Technologies prior to joining FNLCR. Esposito can be reached by email at dominic.esposito@nih.gov.

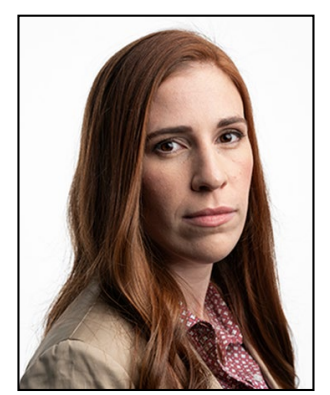

Kaitlyn Sadtler is an Investigator and chief of the Section of Immunoengineering at the National Institute of Biomedical Imaging and Bioengineering (NIBIB) at the National Institutes of Health (NIH). Her lab utilizes classic immunologic techniques to understand the basic mechanistic biology behind immune responses to medical device implants and how to leverage that knowledge to develop nextgeneration immunomodulatory materials for regenerative medicine. She received her $\mathrm{PhD}$ degree from Johns Hopkins University and completed her postdoctoral fellowship at the Massachusetts Institute of Technology prior to joining the $\mathrm{NIH}$. Sadtler can be reached by email at kaitlyn. sadtler@nih.gov. 